\section{$\mathbf{m} / \mathbf{s}$}

médecine/sciences $1998 ; 14: 395-7$

\title{
L'ORIGINE INFECTIEUSE DE CERTAINES MALADIES IDIOPATHIQUES
}

\section{Patrick Berche}

\section{RÉFÉRENCES}

1. Evans AS. Causation and disease. New York, London : Plenum Medical Book Company 1993 : 238.

2. Fredricks DN, Relman DA. Sequence-based identification of microbial pathogens : a reconsideration of Koch's postulats. Clin Microbiol Rev $1996 ; 9: 18-33$.

3. Bode L, Zimmermann W, Ferszt R, Steinbach F, Ludwig H. Bornadisease virus genome transcribed and expressed in psychiatric patients. Nat Med $1995 ; 1: 232-6$.

4. Rettig MB, Mu HJ, Vescio RA, Pöld M, Schiller G, Belson D, Savage A, Nishikubo C, Wu C, Fraser J, Said JW, Berenson JR. Kaposi's sarcoma-associated herpesvirus infection of bone-marrow dendritic cells from multiple myeloma patients. Science 1997 ; 276 : 1851-4.

5. Diener TO. The frontier of life : the viroids and viroids-like satellite RNAs in viroids ans satellites. In : Maramorosch K, ed. Molecular parasites at the frontier of life. Boca Raton, Florida : CRC Press Inc, 1991.

6. Poisson F, Roingeard P, Goudeau A. Le virus de l'hépatite delta : un mode de réplication bien singulier. Med Sci 1995 ; 11 : 1379-87.

7. Conrad B, Weissmahr RN, Böni J, Arcari R, Schüpbach J, Mach B. A human endogenous retroviral superantigen as candidate autoimmune gene in type I diabetes. Cell 1997 ; 90 : 303-13.

8 . Eickbush TH. Origin and evolutionary relationships of retroelements. In : Morse SS, ed. The evolutionary Biology of Viruses. New York : Raven Press Ltd, 1994 : 121-57.

9. Renault S, Bonnin-Rouleux F, Periquet G. Eléments transposables et évolution des génomes. $\mathrm{Vi}$ rologie 1997 ; 1 : 133-44.

10. Reiter LR, Murakami F, Koeuth T, Pentao L, Muzny DM, Gibbs RA, Pupski JR. A recombination hot spot responsible for two inherited peripheral neuropathies is located near a mariner transposonlike element. Nat Genet 1996 ; 12 : 288-97.

\section{ADRESSE ET TIRÉS A PART}

P. Berche : professeur des universités, praticien hospitalier, directeur de l'U. 411 de l'Inserm. Inserm U. 411, Faculté de médecine NeckerEnfants Malades, 156 , rue de Vaugirard, 75730 Paris Cedex 15, France. a Science est née avec le concept de causalité. Tout phénomène a une cause naturelle : le soleil apporte la lumière, le feu provoque la fumée, une blessure induit la douleur. Cette démarche a été appliquée par les Grecs aux maladies : toute maladie a une cause naturelle, la contagion et les épidémies ont des causes. On a donc d'abord classé les maladies selon les symptômes observés, leur localisation, leur caractère contagieux ou leur évolution aiguë ou chronique. Puis, cette classification est devenue anatomoclinique en essayant de relier les symptômes observés aux lésions anatomiques observées à l'autopsie et au cours de l'examen microscopique des tissus. Les découvertes de Pasteur sur les germes ont permis de définir un lien de causalité direct entre certains micro-organismes et des maladies souvent contagieuses. La contagion responsable de mystérieux et incompréhensibles phénomènes épidémiques atteignant l'ensemble du monde vivant, était liée à des germes spécifiques (virus, bactéries ou parasites) responsables de maladies stéréotypées. En quelques décennies, de nombreux agents pathogènes furent ainsi identifiés, permettant d'assigner des causes spécifiques à de nombreuses maladies contagieuses. Le lien de causalité reposait sur les postulats de Koch-Henlé : (1) le microorganisme est constamment associé à la maladie et isolé dans des circonstances qui rendent compte des signes cliniques et des lésions anatomopathologiques (par exemple, ils sont mis en évidence dans les tissus infectés) ; (2) le micro-organisme n'est retrouvé dans aucune autre maladie, excepté de façon fortuite; (3) le micro-organisme isolé peut expérimentalement reproduire la maladie $[1,2]$.

\section{De nouvelles entités nosologiques liées aux micro-organismes}

Tout cela était trop simple. On s'aperçut rapidement qu'un microorganisme donné pouvait entraîner un large spectre de syndromes cliniques, et pouvait même être présent chez des individus en bonne santé mais contagieux. Cela mettait en lumière qu'une maladie infectieuse pouvait avoir plusieurs causes, incluant, outre l'agent infectieux luimême, la sensibilité génétique de l'hôte infecté et des facteurs liés à l'environnement. On prit aussi conscience qu'un même syndrome clinique, par exemple le syndrome grippal, pouvait être déclenché par de multiples agents infectieux autres que le virus de la grippe, Myxovirus influenzae. Cela conduisit à reconsidérer les postulats de Koch-Henlé, souvent impossibles à démontrer (en particulier le $3^{\mathrm{e}}$ postulat), et à faire émerger la notion de risque relatif. Assigner un rôle à un agent infectieux revient à mettre en évidence une forte association entre l'exposition au risque (le micro-organisme) et la distribution spatio-temporelle de la maladie $[1,2]$. Bien sûr, le micro-organisme peut aussi être mis en évidence par culture ou PCR et indirectement par l'apparition d'anticorps spécifiques chez les patients soumis au risque.

Il demeure que de nombreuses maladies idiopathiques d'évolution aiguë, récurrente ou chronique, souvent inflammatoires et apparemment non 
héréditaires, pourraient être d'origine infectieuse. La stratégie pour assigner une cause infectieuse à ces maladies passe par une approche multidisciplinaire, définissant l'entité nosologique, l'exposition au risque (épidémiologie) et cherchant à mettre en évidence le micro-organisme. Comment isoler de la gorge la bactérie responsable de la diphtérie sans circonscrire, au début du XIX ${ }^{\mathrm{e}}$ siècle, au milieu du fatras des angines de l'enfant, une angine pseudomembraneuse maligne souvent associée au croup ? N'en est-on pas à ce stade pour les maladies psychiatriques par exemple, où il semble ne pas exister de définitions nosologiques consensuelles notamment pour les syndromes maniaco-dépressifs ou les dépressions. Ces maladies psychiatriques méritent d'être amplement étudiées sur le plan de l'exposition à certains virus neurotropes. Une polémique existe actuellement sur le rôle des Bornavirus comme causes de syndromes dépressifs et maniacodépressifs chez l'homme [3]. Ces virus sont connus depuis près d'un siècle pour être responsables de graves troubles comportementaux chez de nombreuses espèces animales. On ne les étudie que depuis un peu plus d'une décennie chez l'homme. Définir l'entité nosologique est donc une première étape cruciale. Certaines maladies idiopathiques pourraient donc être des syndromes disparates encore mal définis. Il faut ensuite déterminer l'épidémiologie de la maladie, c'est-à-dire l'exposition au risque qui permet d'évoquer l'origine infectieuse, comme ce fut le cas pour le SIDA en 1979. Il faut chercher à mettre en évidence des pics de fréquence, des concentrations de malades dans certaines régions, à définir avec précision la répartition spatio-temporelle de la maladie et les comportements des patients. Cela permet de faire des prédictions sur les modes de transmission et la nature de l'agent causal. Certaines maladies, notamment la sclérose en plaque, la maladie de Crohn, ou le diabète insulinodépendant, ont des distributions qui peuvent évoquer une exposition à un risque infectieux $\left(\mathrm{m} / \mathrm{s} n^{\circ} 11\right.$,

\section{Mettre en évidence l'agent causal}

Montrer la présence d'un microorganisme ou la persistance de génomes étrangers chez les patients est l'ultime étape, et certainement l'une des plus ardues pour diverses raisons. Tout d'abord, l'agent étiologique peut être un micro-organisme ubiquitaire, largement présent dans la population générale, induisant une maladie "idiopathique " chez certains individus génétiquement prédisposés. Ces agents peuvent être absents ou très difficiles à mettre en évidence au moment du syndrome inflammatoire aigu ou récurrent. Tel est le cas du rhumatisme articulaire aigu, de certaines glomérulonéphrites, ou de la chorée de Sydenham, associés à Streptococcus pyogenes. Cela est aussi vrai pour les manifestations tertiaires de la syphilis, les arthrites postinfectieuses après infection par Yersinia, Salmonella ou Shigel$l a$, ou après infections entériques à Campylobacter jejuni (parfois associé au syndrome de Guillain-Barré $[\mathrm{m} / \mathrm{s}$ $n^{\circ} 2$, vol. 12, p. 262J). Dans le cas des virus, la persistance de certains génomes viraux (herpesvirus, rétrovirus, virus de la rougeole...) est parfois à l'origine de diverses maladies, telles que le lymphome de Burkitt ou le carcinome du rhinopharynx (virus Epstein-Barr $)\left(\mathrm{m} / \mathrm{s} n^{\circ} 12\right.$, vol. 11, p. 1765), la panencéphalite sclérosante subaiguë (virus de la rougeole), la leucoencéphalite multifocale progressive (polyomavirus), certaines leucémies (HTLV-1), le sarcome de Kaposi et le myélome multiple (HHV-8) $(\mathrm{m} / \mathrm{s}$ $n^{\circ} 11$, vol. 11, p. 1605) [4]. La plupart de ces virus infectent le plus souvent la population sans conséquences cliniques, comme en témoignent les études séro-épidémiologiques et la mise en évidence de génomes viraux dans les cellules. Il est donc souvent très difficile de leur assigner un rôle étiologique. Certains de ces germes pourraient être impliqués dans certaines maladies auto-immunes, telles que la polyarthrite rhumatoïde (EBV, CMV), le lupus érythémateux disséminé (EBV), le diabète insulinodépendant (Coxsackie B, rétrovirus), la maladie de Gougerot-Sjögren, les thyroïdites autoimmunes notamment, ou encore dans certaines maladies héma- tologiques (myélomes, leucémies...), certaines maladies inflammatoires chroniques digestives (maladie de Crohn, rectocolite hémorragique...), la sarcoïdose, les maladies démyélinisantes (sclérose en plaques...), certaines épilepsies (encéphalite de Rasmussen [m/s $n^{\circ} 10$, vol. 11, p. 1177], syndrome de West, syndrome de Lennox et Gastaut...), ou même certaines maladies cardiovasculaires comme la maladie de Kawasaki, la maladie de Takayasu ou encore l'athérosclérose (pour laquelle une association avec une bactérie Chlamydia pneumoniae a été récemment évoquée). Le syndrome de fatigue chronique ou certaines morts subites du nourrisson sont à ajouter à cette liste. Qui est l'œuf? qui est la poule ? La difficulté est liée au «trop plein » d'agents infectieux, et la relation de causalité ne peut venir que d'études rigoureuses, cohérentes et convergentes, montrant une forte association entre la maladie et l'exposition à un micro-organisme donné.

\section{Découvrir \\ de nouveaux agents infectieux}

Certaines maladies idiopathiques pourraient aussi être liées à des microorganismes inconnus. Il est ainsi possible d'isoler des virus nouveaux à partir des tissus (HHV-6 et rétrovirus au cours de la sclérose en plaque $[\mathrm{m} / \mathrm{s}$ $n^{\circ}$ 3, vol. 14, p. 372], rétrovirus au cours du diabète insulinodépendant [m/s $n^{\circ} 11$, vol. 13, p. 1362], spumavirus au cours d'un carcinome nasopharyngé, HHV-8 au cours du myélome multiple $\left[\mathrm{m} / \mathrm{s} n^{\circ} 11\right.$, vol. $\left.\left.11, p .1605\right] \ldots\right)$. Le lien de causalité est là aussi difficile à établir et ne peut reposer que sur un faisceau convergent d'arguments épidémiologiques, immunologiques et virologiques. C'est ce qui a été réalisé pour montrer le rôle étiologique de Helicobacter pylori dans l'ulcère duodénal, certaines gastrites et des cancers gastriques $\left(\mathrm{m} / \mathrm{s} \mathrm{n}^{\circ} 4\right.$, vol. 10, p. 480). L'efficacité du traitement antibiotique sur l'ulcère duodénal a apporté une preuve déterminante de l'importance de ce germe au cours de cette maladie «psychosomatique». Cet extraordinaire exemple doit aussi nous inciter à remettre en cause les croyances les plus établies pour certaines maladies. 
Étonnant aussi est le long cheminement qui a permis de démontrer le rôle des prions dans la maladie de Creutzfeldt-Jakob, les arguments décisifs venant des expériences sur les souris transgéniques et knock-out $\left(\mathrm{m} / \mathrm{s} n^{\circ} 8\right.$ 9, vol. 9, p. 989). La découverte de ces protéines de structure anormale, entraînant des "maladies infectieuses » très particulières, car sans réaction inflammatoire, pourrait ouvrir un pan nouveau de la pathologie, en suggérant qu'une protéine de configuration anormale peut être associée ou à l'origine d'une maladie. Nous sommes ici à la limite entre maladie génétique et maladie infectieuse, puisqu'il existe des formes héréditaires de la maladie de Creutzfeldt-Jakob. D'autres maladies ne pourraient-elles pas être liées à de telles anomalies protéiques? Enfin, parmi les agents infectieux de nature nouvelle, il faut citer les viroïdes, petits ARN monocaténaires ( $300 \mathrm{nu}-$ cléotides) non codants, réplicatifs, responsables uniquement chez les plantes de maladies transmissibles [5]. Si l'on accepte l'idée de l'universalité des phénomènes biologiques, il est possible que ces parasites moléculaires puissent infecter les animaux et l'homme. Il existe d'ailleurs un domaine viroïde chez le virus delta de l'hépatite, montrant qu'une telle structure peut exister chez les animaux [6].

Des découvertes récentes éclairent d'un jour nouveau la physiopathologie de maladies inflammatoires idiopathiques. Tout d'abord, la mise en évidence récente du rôle (hypothétique) d'un rétrovirus endogène dans la genèse du diabète insulinodépendant $\left(\mathrm{m} / \mathrm{s} n^{\circ} 11\right.$, vol. $\left.13, p .1362\right)$ [7]. On sait que $10 \%$ du génome humain est formé d'éléments transposables, source de variabilité génétique importante. Ces éléments transposables, à l'instar de certains virus, sont capables de sortir du génome et de se réinsérer dans de nouveaux sites chromosomiques, mais semblent incapables de se propager à d'autres cellules, à la différence des transposons procaryotes $[8,9]$. Certains rétrotransposons dits de classe I synthétisent une transcriptase inverse et possèdent ou non des séquences LTR, d'autres ne codent pas pour une transcriptase inverse et utilisent une transcriptase inverse en trans pour transposer. Les transcriptases inverses des rétrotransposons sont phylogénétiquement apparentées à celles des rétrovirus et hepadnavirus (dont le virus de hépatite B) des mammifères et des oiseaux, des caulimovirus des plantes, et des introns de type II des mitochondries et de certaines enzymes de bactéries. D'autres rétrotransposons, dits de classe II, codent pour des éléments ressemblant aux séquences IS des bactéries qui codent pour des transposases. Ces éléments pourraient jouer un rôle dans la genèse de certaines maladies. Ainsi, il vient d'être montré que le génome des mammifères, incluant l'homme, possédait de nombreux éléments dits mariner, apparentés à ces IS et appartenant à une superfamille très ubiquitaire dans le monde vivant. Deux maladies humaines, la maladie de CharcotMarie-Tooth de type 1A et une neuropathie héréditaire, seraient dues à des crossing-over inégaux sur le chromosome 17 , liées à un élément mariner à l'origine de cette recombinaison aberrante $\left(\mathrm{m} / \mathrm{s} n^{\circ} 6-7\right.$, vol. 12 , p. 833) [10]. L'activité transcriptase inverse des rétrotransposons pourrait aussi concourir à l'intégration d'ARN viral dans les chromosomes. Certains virus à ARN, comme celui de la chorioméningite lymphocytaire qui induit une réponse immunitaire prolongée chez l'animal, seraient « rétrotranscrits» [11]. Le génome de ce virus persisterait ainsi et serait exprimé dans les cellules infectées, induisant la synthèse continue de protéines étrangères chez l'hôte et une réponse immunitaire prolongée [11]. La facilité avec laquelle l'ADN étranger peut pénétrer le noyau et persister, sous forme épisomique ou intégrée partiellement ou totalement, est aussi illustrée par les vaccins d'ADN qui induisent une réponse immunitaire prolongée. Très récemment, il a été même montré que des bactéries à croissance intracellulaire (Salmonella typhimurium) pouvaient être le vecteur de gènes bactériens capables de s'intégrer dans les cellules de l'hôte après infection orale $\left(\mathrm{m} / \mathrm{s} n^{\circ} 4\right.$, vol. $\left.14, p .502\right)$ [12], à l'instar de ce qui a été décrit pour le plasmide de Agrobacterium tumefacians, un pathogène des plantes. Ces transferts horizontaux de gènes viraux ou bactériens aux cellules eucaryotes pourraient avoir une pertinence physiopathologique. Certains résultats obtenus avec des lignées de souris transgéniques illustrent les conséquences pathologiques de l'acquisition d'ADN étranger. L'introduction du gène codant pour la sous-unité Al de la toxine cholérique (une ADP-ribosylase) chez la souris entraîne un gigantisme avec hypertrophie pituitaire et hyperthyroïdie [13]. De même, les souris transgéniques pour un spumavirus sans aucune pathogénicité démontrée, développent des encéphalopathies progressives avec myopathies mortelles en 4 à 6 semaines, alors que l'introduction d'un seul gène transactivateur de ce virus (bel) entraîne une maladie plus lente et plus tardive $\left(\mathrm{m} / \mathrm{s} n^{\circ} 5\right.$, vol. $\left.10, p .596\right)$ [14]. Tous ces exemples illustrent la complexité des mécanismes physiopathologiques qui pourraient être à l'origine de maladies «idiopathiques» et imposent une réflexion sur la notion même d'agent infectieux. N'y a-t-il pas des maladies qui seraient le résultat de l'acquisition de gènes étrangers exprimés chez certains patients, ou entraînant des dysfonctionnements graves par leur localisation chromosomique? Ces maladies forment donc un grand chantier de recherche pour l'avenir avec, en perspective, l'espoir de traitements nouveaux pour les patients

\section{RÉFÉRENCES}

10. Reiter LR, Murakami F, Koeuth T, Pentao L, Muzny DM, Gibbs RA, Pupski JR. A recombination hot spot responsible for two inherited peripheral neuropathies is located near a mariner transposonlike element. Nat Genet 1996 ; 12 : 288-97.

11. Klenerman P, Hengartner H, Zinkernagel RM. A non-retroviral RNA virus persists in DNA form. Nature 1997 ; 390 : 298-301.

12. Darji A, Guzman CA, Gerstel B, Wackholz P, Timmis KN, Wehland J, Chakraborty T, Weiss S. Oral somatic transgene vaccination using attenuated Salmonella typhimurium. Cell 1997 ; 91 : 765-75. 13. Burton FH, Hasel KW, Bloom FE, Sutcliffe JG. Pituitary hyperplasia and gigantism in mice caused by a cholera toxin transgene. Nature 1991 ; 350 : 74-7. 14. Saïb A, Periès J, Dithé H. Recent insights into the biology of the human foamy virus. Trends $M i$ crobiol $1995 ; 3$ : $173-8$.

\section{TIRÉS À PART}

P. Berche. 